provided stool for FC concentration analysis and the study was terminated once the last recruited patient reached a follow up period of 365 days. Remission was defined as a Crohn's disease activity index (CDAI) of $<150$. Relapse was defined as either a need for escalation of medical therapy, surgery for active CD or progression of disease phenotype using the Montreal classification. The study was approved by the West of Scotland Research Ethics Service (REC reference 10/ S0704/1). The Receiver Operating Characteristic (ROC) curve of relapse by 12 months, based on FC value at baseline, was calculated. Kaplan-Meier curves of time to relapse, some of which were longer than 12 months, were based on the resulting best FC cut-off value for predicting relapse (with patients who had not relapsed being censored at end of follow-up) and compared using the log-rank tests.

Results 98 patients were recruited. One patient was lost to follow up, 1 died and the care of 3 patients was transferred to another centre, before either relapsing or being followed up for 12 months. Of the 93 remaining patients 11 (12\%) had relapsed by 12 months. The median FC was lower for non-relapsers, $96 \mu \mathrm{g} / \mathrm{g}$ (IOR 39-237), than for relapsers, $328 \mu \mathrm{g} / \mathrm{g}$ (IOR 189-574), ( $p=0.008)$. The area under the ROC curve to predict relapse using FC was $74.8 \%$ (Figure 1). A cut-off FC value of $240 \mu \mathrm{g} / \mathrm{g}$ to predict relapse of quiescent Crohn's disease over the course of one year was associated with a sensitivity of $72.7 \%$ and specificity of $74.3 \%$. Negative predictive value was high at $95.3 \%$ and positive predictive value was $27.6 \%$. There was a significant difference in time to relapse for those with the first FC value below or above $240 \mu \mathrm{g} / \mathrm{g}$ ( $\mathrm{p}=0.011$ ).

Conclusion In this prospective dataset, FC appears to be a useful, non-invasive tool to help identify quiescent Crohn's disease patients at a low risk of relapse over the ensuing 12 months. A FC value of $240 \mu \mathrm{g} / \mathrm{g}$ was deemed the best cut-off value in our patients.

Disclosure of Interest None Declared.

\section{PTH-079 THIOPURINE WITHDRAWAL FOR SUSTAINED REMISSION IN IBD: A UK MULTICENTRE STUDY}

doi:10.1136/gutjnl-2013-304907.566

$1,{ }^{1 *} \mathrm{~N}$ A Kennedy, ${ }^{2} \mathrm{~S} J$ Reynolds, ${ }^{3} \mathrm{R}$ Dattani, ${ }^{3} \mathrm{H}$ Nayee, ${ }^{4} \mathrm{R}$ Felwick, ${ }^{4} \mathrm{R}$ Harris, ${ }^{5} \mathrm{~S}$ M Senanayake, ${ }^{6} \mathrm{H}$ Al-Hilou, ${ }^{7} \mathrm{D}$ R Gaya, ${ }^{6} \mathrm{P}$ Irving, ${ }^{5} \mathrm{M}$ Parkes, ${ }^{4} \mathrm{~J}$ F R F Cummings, II D Arnott, 1J Satsangi, ${ }^{2} \mathrm{~A}$ Lobo, ${ }^{3} \mathrm{~J} 0$ Lindsay, ' $\mathrm{C}$ W Lees. 'Gastroenterology, Western General

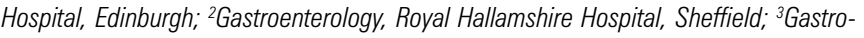
enterology, Barts and the London Hospital, London; ${ }^{4}$ Gastroenterology, Southampton General Hospital, Southampton; 'Gastroenterology, Addenbrooke's Hospital, Cambridge; 'Gastroenterology, Guy's and St Thomas', London; 'Gastroenterology, Glasgow Royal Infirmary, Glasgow, UK

Introduction Thiopurine therapy is effective in maintaining clinical remission in IBD. However, long-term therapy is associated with an increased risk of lymphoma; therefore in clinical practise it may be appropriate to withdraw thiopurines after prolonged remission. Nevertheless, many patients will experience disease relapse within 12 months of drug withdrawal.

The Aim of the present study was to retrospectively determine the relapse rate in ulcerative colitis (UC) and Crohn's disease (CD) following azathioprine (AZA) or mercaptopurine (MP) withdrawal and to determine factors predictive of relapse.

Methods Patients were identified by electronic case note review of IBD patients in eight major centres around the United Kingdom. Major inclusion criteria were AZA and/or MP therapy for a minimum of 3 years, AZA/MP withdrawn due to sustained clinical remission no steroid therapy for 6 months prior to drug withdrawal, and minimum 12 months follow-up.

The primary outcome was disease relapse requiring AZA reinitiation, steroids or colectomy within 12 months of AZA/MP withdrawal, with secondary outcome assessed at 24 months. Clinical/ laboratory predictors of relapse were sought.

Results Data was obtained on 97 patients with CD and 78 with UC. Median age at diagnosis was $26 y$ (interquartile range [IOR] 20-38), and $49 \%$ were female. Median duration of thiopurine use was 73 months (IOR 54-104). Median duration of follow-up was 39 months (IOR 24-65 months).

$\mathrm{CD}$ was associated with a significantly higher risk of relapse than UC on Kaplan Meier analysis (Figure 1, $\mathrm{p}=0.024$ ). The moderatesevere relapse rate for 12 months was $27 \%$ for CD and $14 \%$ for UC. For 24 months, relapse rates were $41 \%$ for CD and $28 \%$ for UC. Elevated CRP was predictive of relapse at 12 months for CD $(0=0.017)$, while elevated platelet count was predictive of relapse at 24 months for UC (0.021).

Retreatment with a thiopurine after relapse was successful in $34 / 39$ (87\%) for CD and 17/18 (94\%) cases for UC.

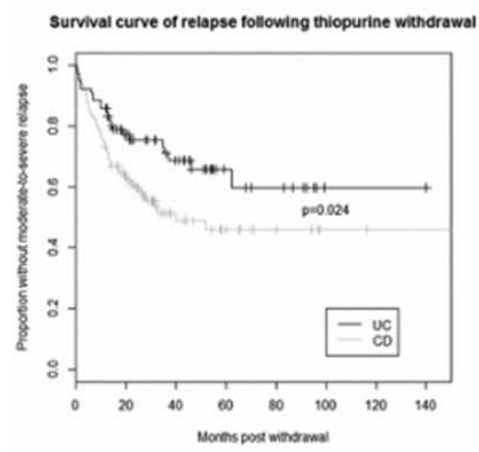

Abstract PTH-079 Figure 1

Conclusion Relapse rates after withdrawal of a thiopurine are high, particularly for $\mathrm{CD}$, and predicting this remains difficult. The findings regarding CRP and $\mathrm{CD}$ in this data highlight the importance of ensuring patients are in deep remission prior to drug withdrawal. Further studies should evaluate the role of faecal calprotectin in this.

Disclosure of Interest None Declared.

\section{PTH-080 DO WE NEED TO SCREEN OUR INFLAMMATORY BOWEL DISEASE (IBD) PATIENTS FOR DEPRESSION: THE PREVALENCE AND SEVERITY OF DEPRESSION WITHIN A TYPICAL DISTRICT GENERAL COHORT OF IBD PATIENTS}

doi:10.1136/gutjnl-2013-304907.567

1," $\mathrm{N}$ Swart, ${ }^{1} \mathrm{D}$ Wellsted, ${ }^{2} \mathrm{~K}$ Lithgo, ${ }^{2} \mathrm{~T}$ Price, ${ }^{2} \mathrm{M}$ W Johnson. ${ }^{1}$ Centre for Lifespan and Chronic Illness Research, University of Hertfordshire, Hatfield; ' ${ }^{2}$ Gastroenterology, Luton \& Dunstable University Hospital, Luton, UK

Introduction Depression is believed to occur in 15 to $30 \%$ of IBD patients, in which suicide is not an uncommon ideation. Some researchers believe that psychiatric illness may have an aetiological role to play in the onset of inflammatory bowel disease (IBD), as the incidence of depression seems to be concentrated in the year before and after the initial diagnosis is made.

Objectives To assess the true prevalence and severity of depression within our inflammatory bowel disease patients.

Methods 2400 patients with IBD in the Luton \& Dunstable catchment were invited to participate in a web-based quality of life assessment, with the option to request a paper copy. All patients were deemed eligible provided they were over 18 and under 90 years of age, with no major learning difficulties or pre-existing serious mental disorders. The well validated 9-item self-report "Patient Health Questionnaire" (PHO) was used. The PHQ-9 has a minimum possible score of 0 and a maximum possible score of 27 . Scores of $5,10,15$, and 20 represent cut-off scores for mild, moderate, moderately severe, and severe depression.

Results 245 patients completed the assessment (43\% male; mean age $=53, \mathrm{SD}=17) .45 \%$ had Ulcerative Colitis, $45 \%$ had Crohn's 
Disease and 10\% had an alternative form of IBD (e.g. Proctitis, Lymphocytic Colitis or Collagenous Colitis). The ethnic mix in the responding cohort was 91\% Caucasian, $6 \%$ Asian, 2\% Mixed and 1\% was not stated. The sample had a mean score of $7.8(\mathrm{CI}=7-8.6)$. $98(40 \%)$ of patients' scores reflected "no depression"; 64 (26\%) reflected "mild depression"; 33 (14\%) reflected "moderate depression"; 36 (15\%) reflected "moderately severe depression"; 12 (5\%) of scores reflected "severe depression".

Conclusion $20 \%$ of our responding IBD patients were shown to have clinically significant levels of depression (moderately severe + severe), with $5 \%$ demonstrating scores suggestive of severe depression ( $1 \%$ expressing suicidal ideation). Relapse rates are known to be closely correlated with the severity of depression, and yet very few are on active treatment or review for this. The prevalence and severity of depression in our cohort of responding IBD patients supports the argument for screening all new IBD patients in order to optimise clinical well-being and treatment efficacy.

Disclosure of Interest None Declared.

\section{PTH-081 UNEARTHING THE TRUE PREVALENCE OF ANXIETY WITHIN A TYPICAL DISTRICT GENERAL COHORT OF INFLAMMATORY BOWEL DISEASE PATIENTS: IS IT TIME WE CONSIDERED ROUTINELY SCREENING FOR ANXIETY?}

doi:10.1136/gutjnl-2013-304907.568

1,"N Swart, 'D Wellsted, ${ }^{2} \mathrm{~K}$ Lithgo, ${ }^{2 \mathrm{~T}}$ Price, ${ }^{2} \mathrm{M}$ W Johnson. ${ }^{1}$ Centre for Lifespan and

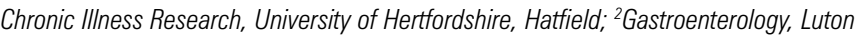
\& Dunstable University Hospital, Luton, UK

Introduction Previous studies have suggested that 15 to $30 \%$ of inflammatory bowel disease (IBD) patients also suffer from anxiety. Whilst most gastroenterologists would feel confident in recognising and diagnosing florid steroid induced psychosis, much of the associated anxiety experienced by IBD patients goes undiagnosed and untreated. Disease severity, recurrent flares, poor treatment adherence, disability, unemployment status, and socio-economic deprivation are all believed to be associated with anxiety in these patients. The severity of anxiety also appears to be directly correlated to the physical morbidity and malnutrition risk.

Objectives To assess the true prevalence of anxiety within our IBD patients.

Methods 2400 patients with IBD in the Luton \& Dunstable catchment were invited to participate in a web-based quality of life assessment, with the option to request a paper copy. Eligibility criteria required patients to be between 18 and 90 years of age, with no serious learning difficulties or pre-existing serious mental disorders. The well validated 7-item self-report "Generalised Anxiety Disorder (GAD) Questionnaire" was used. The GAD-7 has a minimum possible score of 0 and a maximum possible score of 21 . Scores of 5,10 , and 15 represent cut-off scores for mild, moderate, and severe anxiety.

Results 245 patients completed the assessment (43\% male; mean age $=53, \mathrm{SD}=17$ ). $45 \%$ had Ulcerative Colitis, $45 \%$ had Crohn's Disease and $10 \%$ had an alternative form of IBD (e.g. Proctitis, Lymphocytic Colitis, or Collagenous Colitis). The ethnic mix in the responding cohort was $91 \%$ Caucasian, $6 \%$ Asian, 2\% Mixed and 1\% was not stated. The sample had a mean score of $6.6(\mathrm{CI}=5.9-7.4)$. $72 \%$ of patients' scores reflected no anxiety or "mild anxiety"; $15 \%$ reflected "moderate anxiety"; and 13\% reflected "severe anxiety".

Conclusion $29 \%$ of our responding IBD patients were shown to have significant anxiety scores (moderate + severe), with 14\% demonstrating severe anxiety levels. Despite the severity, few of these patients were receiving treatment or therapy for their condition. The GAD score is a simple and quick tool that can be used in clinic. Given that anxiety is believed to directly affect the clinical course of $\mathrm{IBD}$, both the screening and treatment of this condition should be considered part of standard IBD medical care.

Disclosure of Interest None Declared.

\section{PTH-082 SERUM CALPROTECTIN: A NOVEL BIOMARKER TO PREDICT OUTCOME IN ACUTE SEVERE ULCERATIVE COLITIS?}

doi:10.1136/gutjnl-2013-304907.569

1." $\mathrm{N}$ C Hare, ' N A Kennedy, ${ }^{1} \mathrm{~K}$ Kingstone, 'I D Arnott, ' ${ }^{1} \mathrm{~A}$ G Shand, ${ }^{1} \mathrm{~K}$ R Palmer, ${ }^{1} \mathrm{D}$ Penman, ' $\mathrm{C}$ W Lees, '1 Satsangi. 'Gastrointestinal Unit, University of Edinburgh, Western General Hospital, Edinburgh, UK

Introduction Acute severe ulcerative colitis (ASUC) remains an important clinical problem and is associated with significant morbidity and requirement for colectomy. Faecal calprotectin and C-reactive protein (CRP) have previously been shown to predict the need for colectomy but there is an unmet need for further biomarkers. Serum calprotectin has not previously been analysed for this purpose and may provide a novel way of determining disease activity and outcome. The aim of this study was to assess how serum calprotectin relates to faecal calprotectin and other blood markers of inflammation, and to determine whether serum calprotectin on admission predicts colectomy.

Methods Blood samples were collected prospectively from patients who presented with ASUC as defined by the Truelove and Witts criteria. Blood samples were taken within the first 24 hours of admission. Faecal samples were pre-processed using $\mathrm{PhiCal}^{\mathrm{TM}}$ extraction buffer. Samples were stored at $-80^{\circ} \mathrm{C}$ and analysed in duplicate using the PhiCal ${ }^{\mathrm{TM}}$ calprotectin ELISA according to manufacturer's instructions. Samples with a calprotectin result of $>2500 \mu \mathrm{g} / \mathrm{g}$ were diluted and retested. Statistical comparisons were made between serum calprotectin and other markers of inflammation using Spearman's correlation coefficient, and ROC curve analysis was performed to determine how well each test performed in predicting colectomy.

Results There were 45 patients recruited to the study with ASUC, of which $22(49 \%)$ were female. Median age on admission was 40 years (interquartile range [IOR] 26-62). Median disease duration was 12 years (IOR 0-59). 26 of the 45 patients had a paired faecal sample for calprotectin analysis. There was no difference in sex, age or disease extent between those with or without faecal calprotectin. Serum calprotectin correlated significantly with CRP $\left(\mathrm{R}^{2}=0.46\right.$, $\mathrm{p}<0.0001)$ and with albumin $\left(\mathrm{R}^{2}=0.12, \mathrm{p}=0.023\right)$ but not with faecal calprotectin $\left(R^{2}=0.02, p=0.450\right)$. ROC analysis gave an AUC of 0.69 for serum calprotectin compared with 0.71 for CRP and 0.58 for faecal calprotectin. A cut off for serum calprotectin of $400 \mu \mathrm{g} / \mathrm{g}$ gave a sensitivity of 0.68 and a specificity of 0.69 .

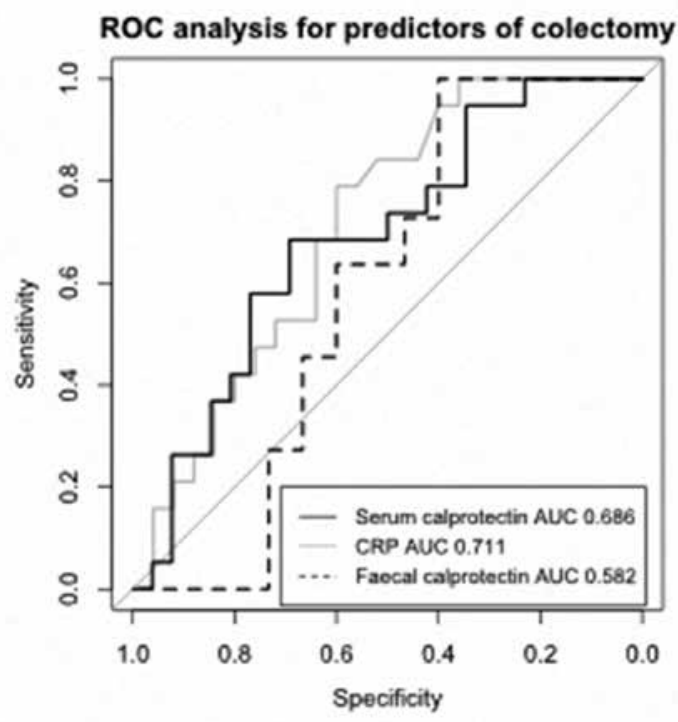

Abstract PTH-082 Figure 1 